\title{
ADDENDA FROM PRE-MENINSKI TRANSCRIPTION TEXTS TO STANISŁAW STACHOWSKI'S “OSMANLI TÜRKÇESINDE YENI FARSÇA ALINTILAR SÖZLÜĞÜ". PART VII
}

Keywords: Ottoman-Turkish, New Persian, lexical borrowing, transcription texts

\begin{abstract}
Stanisław Stachowski wrote a series of articles devoted to studies on the New Persian loanwords in Ottoman-Turkish, which were published in Folia Orientalia in the 1970 and later republished in 1998 as a single volume. Since then, however, a good number of editions of new Ottoman texts have appeared, especially transcription texts dating from before Meninski's Thesaurus (1680), which provide much new lexical material. Within this material there are many Persianisms - predictably enough where Ottoman-Turkish is concerned. This paper aims to supplement Stachowski's work with words of Persian origin taken from pre-Meninski transcription texts. It is divided into two parts, the first including data to be added to entries already recorded by Stachowski (eight articles), the second containing data that constitute new entries (three articles). A short historical-etymological note on the words dealt with also features at the end of each entry.
\end{abstract}

452. $\operatorname{raf}(1680)$ - 1650 raf (raf) 'ripostiglio, credenza' (CarrR. 276).

- < Pers. raf'seat, bench, or settle at a house-door; moulding, tablet (...) serving as an ornament to a room, or as a shelf'. - N. 511 (XV), P. (-).

453. raht (räht 1680); rat (ca. 1630) - ca. 1630 rat (rat) 'frenum equi' (MontR. 165); 1641 raht (raht) 'fornimento' (MolDitt. 152); 1677 raht (raht) 'fornimento di cauallo' (MascVoc. 52).

Phr. - 1677 at rahtı (at rahti) 'adornamento di cau[a]llo' (MascVoc. 7). 
- 1677 rahtı çıkar- (rahti ciccarmach) 'sbrigliare', (rahti ciccarmisc) 'sbrigliato', (rahti ciccaran) 'sbrigliatore' (MascVoc. 181).

- < Pers. raxt 'goods and chattels, apparatus; housings, harness'. - N. (-); P. 204 (XIII/XIV).

454. rast (1680; rast gel-1641, ras ol-1672); rasit (1650) - 1533 rast (raást) 'appunto, pari' (ArgAd. 250, ArgR. 201).

Der. - 1533 rastla- (raastlárum) 'pareggio, ragguaglio, fo pari una cosa', (raastlatterúrum) 'fo pareggiare, fo ragguagliare' (ArgAd. 250, ArgR. 201).

Phr. - 1650 rasit et- (rasit ederum) 'giustare, agguagliare, pareggiare' (CarrR. 277) - 1533 rast gel- (rast ghielúrum) 'riuscire una cosa come tu disegnaui, uenire appunto' (ArgAd. 250, ArgR. 201); 1567 rast gel- (rasst gelŭp) 'ci siamo imbattuti' (LettBomb. 140-144); 1611 ras gel- (rasgelmes) 'decente' (RJTMajd. 45); 1611 ras gel- (rás ghielérum) 'affrontare, scontrare; imbattersi, incontrare' (FerrR. 132); 1677 ras gel- (ras ghielmech) 'affrontare; imbattersi, scontrare, incontrare', (ras ghielmisc) 'affrontato, scontrato' (MascVoc. 7, 62, 191) - 1650 rasit ol- (rasit olmisc) 'giustato, pareggiato' (CarrR. 277).

- < Pers. rāst 'right, true; good, just, sincere, upright; straight, even, level'. N. 513 (XII); P. 205 (XIII/XIV).

456. râvent (revent 1641) - 1533 revent (reuént) 'reubarbaro, riobarbaro' (ArgAd. 251, ArgR. 203).

- < Pers. rāwand 'rhubarb'. - N. 514 (XIII); P. (-).

457. rençper (irençper 1544/48); renşper (ca. 1630) - 1533 rençper (rencipér) 'affaticante, uno che uiue delle braccia' (ArgAd. 250, ArgR. 202); 1575 rençper (rentsper) 'ouurier; calo' (PostelInstr.); 1611 rençper (rencipér) 'faticatore, lauorante, operario' (FerrR. 132); ca. 1630 renşper (rensper) 'operarius' (MontR. 165).

Der. - 1533 rençperlük (renciperlúch) 'l'arte sua (scil. di uno che vive delle braccia)' (ArgAd. 250, ArgR. 202); 1611 rençperlik (renciperlíc) 'fatica, opera quotidiana, opera' (FerrR. 132).

Phr. - 1611 rençperlik et- (renciperlíc edérum) 'faticare' (FerrR. 132).

- < Pers. ranğ-bar 'artificer, mechanic'. - N. 518 (XV); P. (-).

458. rende (1641); rendele (1587/88), erende (1611) - 1533 rende (rendé) 'pialla' (ArgAd. 250, ArgR. 202); 1587/88 rendele (rendele) 'Hobel' (LubAd. 54); 1611 erende (erendé) 'chiana, instrumento di mastro d'ascia; gratta caso' (FerrR. 132); 1650 rendele (rendele) 'pialla da legnaiolo' (CarrR. 278).

Der. - 1533 rendecik (rendeggích) 'pialletto' (ArgAd. 250, ArgR. 202).

- 1533 rendele- (rendelérum) 'piallo', (rendeletterúrum) 'fo piallare' (ArgAd. 250, ArgR. 202); 1650 rendele- (rendelerum) 'grattar formaggio; piallare, pianare', (rendelemech) 'piallamento', (rendelenmisc) 'piallato, pianato' (CarrR. 278) - 1650 rendeleci (rendelegi) 'piallatore' (CarrR. 278).

- < Pers. randa ‘joyner’s plane’. - N. 518 (XVII Meninski); P. (-). 
459. renk (1603) - 1533 renk (rench) 'colore' (ArgAd. 250, ArgR. 202); 1574 rengi (renghi [+ poss.]) 'colore' (VNAd. 67); 1587/88 reng (reng) 'Farb' (LubAd. 55); 1584 rengi (renghi [+ poss.]) 'couleur' (PalPD. 528-529; [*brachi (!) (PalBern. 321)]); 1611 rengi (renghí [+ poss.]) 'colore' (FerrR. 132); ca. 1630 renk (renk) 'color' (MontR. 165); 1650 ireng (irengh) 'colore' (CarrR. 190).

Der. - 1533 renkli (renchlí) 'colorato' (ArgAd. 250, ArgR. 202) - 1677 irenkisiz (irenchisis) 'pallido, scolorito' (MascVoc. 116).

- 1611 renkle- (renklemek) 'colorear' (RJTMajd. 218); 1650 irengle- (irenghlerum) 'colorire' (CarrR. 190) - 1650 irengleci (irenghlegi) 'coloritore' (CarrR. 190).

Phr. - 1650 irengsiz ol- (irenghsis olurum) 'scolorirsi' (CarrR. 190).

- < Pers. rang 'colour, hue'. - N. 518 (XIII); P. 207 (XIII).

461. rezene (1603); rezenä (1611), razıyane (1641) - 1533 rezene (resené) 'finocchio' (ArgAd. 251, ArgR. 203); 1611 rezenä/raz(i)yane (reszená, rasziané) 'finocchio, herba' (FerrR. 132); ca. 1630 rezene (resene) 'foeniculus' (MontR. 165); 1641 razıyane (raseiane) 'finocchio' (MolDitt. 148); 1677 raziyane (rasiane) 'finocchio' (MascVoc. 50).

- < Pers. rāzıyāna 'fennel'. - N. 521 (XIII); P. 205 (1/XV).

462. rig (1680); ${ }^{\star}$ eril (1533) - $1533{ }^{\star}$ eril [probably to be amended to erig $^{\star} /$ erik $^{\star}$ ] 'poluere d'oro che si mette in su le lettere' (ArgAd. 180, ArgR. 95); 1611 rik (ríc) 'arena' (FerrR. 133).

- < Pers. rigg 'sand, gravel, dust; kind of gold-coloured dust, with which they sprinkle writings'. - N. (-); P. 210 (2/XV).

463. rigdan (1680); rihtan // rihtan (ca. 1630) - 1611 rikdan (ricdán) 'arenaloro' (FerrR. 133); ca. 1630 rihtan // rihtan (rihtan) 'clepsydra' (MontR. 166).

- < Pers. rìgdān 'sand-box'. - N. (-); P. (-).

467. ruşen (1680); ruşan (1533), uruşen (1533) - 1533 ruşan/uruşen (ruscián, uruscén) 'piaceuole, sereno' (ArgAd. 251, 279, ArgR. 250).

- < Pers. rušan 'clear; light, splendid, bright, serene'. - N. (-); P. 211 (1445).

468. ruz (1672) - 1641 ruz (rus) 'tempo' (MolDitt. 449).

- < Pers. rūz 'day; day-time’. - N. 526 (XII); P. 212 (XIII/XIV).

469. ruzname (1668); roznama $^{\star}(1533)$, rozuname* $(1539)$, rozanama $^{\star}$ (1560), rurzname (?) (1611) - 1611 rurzname (?) (rursnamé) 'filosophia' (FerrR. 133); 1612 ruzname (rusname) 'calendarium' (MegILT.); ca. 1630 ruzname (rusname) 'kalendarium' (MontR. 167).

Der. - 1533 roznamacı (rosnamaggí) 'scriuano che tiene conto de' lauori del signore giornalmente’ (ArgAd. 251, ArgR. 203); 1539 “doi Rosunamegi, capi de Scriuani" (RambLibT. 17r); 1560 "Rosanamagi, chef des cõtrerolleurs, qui mettent les deniers dans les trezors” (PostelTPart. 68-69).

- < Pers. ruznāma 'a daily account-book or journal'. - N. 526 (XVII Meninski); P. (-). 
470. rüsvay (1680); rüsfay (1533), rosfay // rösfay (ca. 1630), risfa // rısfa (ca. 1630), rosfa // rösfa (ca. 1630), risvay // rısvay (1641), risfay // rısfay (1677) - 1533 rüsfay (rusfái) 'uituperio di parole' (ArgAd. 251, ArgR. 204); 1611 rüsfay (rusfái) 'bruttamente' (FerrR. 133); ca. 1630 rosfa // rösfa (rosfa) 'vituperium' (MontR. 166); 1650 risvay // rısvay ile (risuai ileh) 'suergognatamente' (CarrR. 278).

Der. - 1533 rüsfaylık (rusfáilich) 'uituperio di parole’ (ArgAd. 251, ArgR. 204); 1677 risfayluk// rısfayluk (risfailuch) 'suergognarsi' (MascVoc. 236).

Phr. - 1533 rüsfay et- (rusfái edérum) 'uitupero', (rusfái etterúrum) 'fo uituperare' (ArgAd. 251, ArgR. 204); ca. 1630 risfa // risfa et-, rosfay // rösfay et- (risfa etmek, rosfai etmek) 'vituperare' (MontR. 166); 1641 risvay // rısvay et- (risuai etmek) 'imbrattare, sporcare, suergognare' (MolDitt. 192, 441); 1650 risvay / risvay et- (risuai ederum) 'deturbare, imbrattare, sporcare', (risuai etmech) 'imbrattamento' (CarrR. 278); 1677 risfay // risfay et- (risfai etmech) 'imbrattare; suergognare' (MascVoc. 62, 236) - 1650 risvay // risvay edici (risuai edigi) 'deturbatore' (CarrR. 278) - 1567 rüsfay ol- (rŭsfay olup) 'se la sarebbero cavata così (indenni)' (LettBomb. 140-144); 1650 risvay // rısvay ol- (risuai olurum) 'imbrattarsi', (risuai olmisc) 'deturbato' (CarrR. 278); 1677 risfay // risfay ol- (risfai olunmisc) 'imbrattato; suergognato' (MascVoc. 62, 236).

- < Pers. ruswāy 'dishonoured, disgraced, infamous, ignominious'. - N. 527 (XIII); P. 213 (1430).

471. rüzgâr (ruzgar [or rather rüz-] 1603); rüzger (1611), ürüzger (1615), örüzgâr/ örüzger (1641) - 1611 rüzger (rusger, rusgèr) 'uiento, l'area [= aria]' (RJTMajd. 219); 1611 rüzger (rusghiér) 'vento’ (FerrR. 133); 1615 ürüzger (vruzghier) 'wind' (PaszkStach. 48); ca. 1630 ürüzger (vrusghier) 'ventus' (MontR. 199).

Phr. - 1641 batı örüzgârı (bati orusghiari) 'garbino, vento' (MolDitt. 159) - 1641 gün dog̀ıı örüzger (ghiun doghisi orusghier) 'leuante, vento' (MolDitt. 233) - 1611 karş(l)lı rüzger (carsclí rusghiér) 'vento contrario' (FerrR. 133) - 1611 kulay rüzger (culái rusghiér) 'vento fauoreuole' (FerrR. 133) - 1615 pek ürüzger (pekuruzgier) 'gale' (PaszkStach. 48).

- 1641 örüzgâr(l) geç- (orusghiari ghieccimek) 'trapassare il vento' (MolDitt. 462); 1677 rüzigâr(i) geç- (rusighiari ghieccimech) 'trapassare il vento' (MascVoc. 253).

- < Pers. ruzgār 'world; time, season; wind, air'. - N. 527 (XII); P. 212 (XIII/XIV).

472. sade (1641).

Phr. - 1533 katife sade (chatifé sadé) 'uelluto stietto et semplice' (ArgAd. 213, ArgR. 141). - $\rightarrow$ 778. diba

- 1677 sade bir katlı (sade bir catli) 'scempio, semplice, non doppio' (MascVoc. 198).

- < Pers. sāda 'pure, unmixed, simple'. - N. 529 (XIV); P. 214 (1445).

473. sahtiyan (1603); saktan (ca. 1520), sanktiyan (?) (1525/30), saktiyan (1574?; ca. 1635), sihtan (1611), satiyan (1611) - ca. 1520 saktan (sactan) 'cordoani' (LupisON. 3a); 
1525/30 sanktiyan (?) (sanctian) 'cordoani' (ITSprAd. 223); 1533 sahtiyan // saktiyan (sacttián) 'cordouano' (ArgAd. 254; ArgR. 207); 1574 sahtiyan // saktiyan (sachtian) 'corame' (VNAd. 67); 1587/88 sahtiyan (sachtian) 'Leder' (LubAd. 55); 1611 sihtan (sichtan) 'cordouan' (RJTMajd. 222); 1611 satiyan (sattián) 'cordoana, pelle' (FerrR. 135); ca. 1635 saktiyan (saktian) 'corium caprinum' (MontR. 170); 1668 satiyan (szattian) 'coreum' (IllNém. 194).

- < Pers. saxtiyān 'goats' leather, morocco'. - N. 531 (XIII); P. 215 (1445).

475. samur (1603) - 1533 samur (samúr) 'zibellino' (ArgAd. 254, ArgR. 209); ca. 1630 samur (samur) 'mus Ponticus, zabellini' (MontR. 170); 1668 samur (szamur) 'pillis czubelina' (IllNém. 193); 1677 samur (samur) 'zibellino, animaletto' (MascVoc. 279).

- < Pers. sammūr 'the Scythian weasel or marten, the sable, also the fine skin or fur of that animal'. The Persian origin of the word is defended also by Doerfer (TMEN 3: 266-267), contrary to the opinion of other scholars (see ErenTDES 355). - N. 535 (XIV); P. (-).

476. saraçhane (1672); serayana (1646) - 1646 "Serajana, gdzie robią siodła, uzdy, strzemiona, i wszytkie inne potrzeby do ubrania konia na wojny" (Sz. Starowolski: StachSHET. 509-510).

- < Pers. sarrāğ-xāna 'a saddler's shop'. - N. (-); P.

478. saray (1546) - [Various adaptations of the word such as It. serraglio, Fr. serrail have not been taken into account] - 1533 saray (sarái) 'palazo' (ArgAd. 255, ArgR. 210); 1545 "proferiscono il Serraglio in loro lingua Sarrai” (BassR. 78); 1557 seray (seraju [Pol. gen.]) ['pałac'] (E. Otwinowski: StachSHET. 499); 1574 saray $^{\star}{ }^{*}$ saiai) 'pallazzo' (sic) (VNAd. 54); 1587/88 saray (saraij) 'ein Palatium oder Palast' (LubAd. 55); 1608 Saraia [possibly = sarayı, + poss.] (SchwSt. 244); 1611 saray (sarrai) 'palazzio' (sic) (RJTMajd. 220); 1611 seray (serái) 'chiostro, palaggio' (FerrR. 136); 1614 “Questa voce 'serraglio' (...) vien così corrotta dalla parola serai, che in lingua di costoro significa propriamente palazzo" (DValCard. 100); ca. 1630 saray (sarrai) 'palatium' (MontR. 171); 1641 saray (sarai) 'chiostro, serraglio, palaggio, palazzo' (MolDitt. 89, 289); 1650 seray (serai, serrai) 'casa, palazzo, serraglio' (CarrR. 289); 1677 seray (serai) 'palagio, palazzo, serraglio' (MascVoc. 116, 200).

Phr. - 1654 "le quatrième [aga] ne sort jamais de Constantinople, \& pour cette raison il s'appelle Seray Aghasi, l'Aga du Serrail” (du Loir: ArvAdd. 467) - 1608 "Vber den Keyserlichen Palast seyn zween obriste Hofmeister /der eine wird genennt Saraia Wascha [= saray başa]" (SchwSt. 244) - 1677 seray naziri (serai nasziri) 'soprastante al palazzo' (MascVoc. 214) - $\rightarrow$ 224. kâhya.

- 1677 kiral saray(l) (chiral sarai) 'palazzo del re' (MascVoc. 120) - $\rightarrow 378$. padişahi.

- < Pers. sarāy 'house, palace, grand edifice, king's court, seraglio'. - N. 538 (XIII); P. 215-16 (1291-1312). 
479. saraydarbaşı (1546) - 1539 "Il Saraidarbassi eunuco del serraglio quãdo il Signor è fuori” (RambLibT. 14v).

- < Pers. sarāy-dār 'garrison-soldier; keeper of the palace' + T. başı. The Turkish phrase has (re)entered into Persian: sarāydār-bāšs 'the majordomo of the palace' (Steingass). - N. (-); P. (-).

480. sarhoş (1603); sorhos // sorhoş (1574), sarkos (1584), sarhos/sarhoç (1611) - 1533 sarhoş (sarchósc) 'jmbriaco, ubbriaco' (ArgAd. 255, ArgR. 210); 1574 sorhos // sorhoş (sorchos) 'imbriacho' (VNAd. 67); 1584 sarkos (sarcos) 'yvrongne' (PalPD. 546-547; missing in PalBern.); 1611 sarhos (sarhòs) 'borracho' (RJTMajd. 52); 1650 sarhoş (sarhhosc) 'briaco' (CarrR. 286); 1668 "Sultan Selim, surnamed *Sarhose [recte Sarhosc], or the drunken" (RycautPSt. 114).

Der. - 1611 saravoşllk (sarauosclíc) 'briachezza' (FerrR. 136); 1650 sarhoşlık (sarhhosclich) 'briachezza' (CarrR. 286).

Phr. - 1533 sarhoş et- (sarchósc edérum) 'fo imbriacare' (ArgAd. 255, ArgR. 210); 1650 sarhoş et- (sarchosc ederum) 'imbriacare altri' (CarrR. 286) 1533 sarhoş ol- (sarchósc olúrum) 'jmbriacomi' (ArgAd. 255, ArgR. 210); 1587/88 sarhoşol-* (or perhaps sarhoşla-*?) (sarhosch *damak) 'vol werden' (LubAd. 15); 1611 sarhoç ol- (sarhotsch olmak) 'borrachear' (RJTMajd. 42); 1650 sarhoş ol(sarchosc olurum) 'imbriacarsi' (CarrR. 286).

- < Pers. sar-xwuš 'merry-headed; tipsy'. - N. 538 (XIV); P. 216 (XIV).

483. sāyvān ( ${ }^{*}$ sevvan ca. 1450); seyvan (1496/1501) - 1496/1501 seyvan (sejwan [with variae lectiones]) ['stan, namiot'] (Constantine of Ostrovica: StachSHET. 506).

- < Pers. sāyabān 'a canopy, umbrella, parasol; tent, pavilion'. - N. 541 (XIV); P. 216 (XIV).

484. saz (1641) - 1533 saz (sas) 'il suono' (ArgAd. 256).

Phr. - 1650 sazeng (= saz ahenk) (sazengh) 'harmonia' (CarrR. 287).

-< Pers. sāz 'musical instrument; concord, harmony'. - N. 541 (XIV); P. 216 (1445).

485. sazende (1641) - 1533 sazende (sasendé) 'sonatore' (ArgAd. 256, ArgR. 212); ca. 1630 sazende (sasende) 'pulsator fidium' (MontR. 172).

- < Pers. sazanda 'player on musical instruments'. - N. (-); P. (-).

490. segbān (seğmen 1591); seymem (seğmem)/semem (1538), sehmen (1539), sekmen (1560), segmen (1630), seyman* (1634), segan (1672) - 1548 "ducẽto huomini chiamati ${ }^{\rtimes}$ Scimi (recte Seim(in)i, It. pl.?) (..) chi di loro gouerna cani grossi, \& chi bracchi \& chi leurieri, \& vanno sempre ad caccia col Signore" (MenTratt. 165); 1634 seyman $^{*}$ (Sejmanowie [Pol. pl.]) (Sz. Starowolski: StachSHET. 505); 1668 "a Militia called Segbans [Engl. pl.], to whose custody the charge of the baggage belonging to the Horse is committed" (RycautPSt. 203); 1672 segban (segbani [Lat. pl.]) 'equites, qui equitatùs impedimenta custodiunt' (HarsColl. 292). 
Phr. - 1538 "Li cacciattori (sic) sonno mille et hanno uno Aga adimandato Seimembassi (elsewhere Semembassi), cioè capo delli cacciatori" (SpandSath. 218); 1539 "vno sechmenbassi capo delli cani da cacciare" (RambLibT. 18r); 1548 "loro (= of the seimi) Capitano chiamato Semibascia" (MenTratt. 165); 1560 "Secmenibaßi, ou maistre Veneur, ou des Veneurs, \& gouuerneurs de chiens" (PostelTPart. 17); 1630 segmen başa ((za) segmen baszą) ['naczelnik segmenów (sejmenów)'] (A. Piaseczyński: StachSHET. 504); 1668 “Segban baschi, the overseer of the carriage of the soldiers baggage" (RycautPSt. 194); 1672 segan başı (seganbasi) 'qui curruum omnium, qui impedimenta, victum, aliaque necessaria instrumenta Jenicseriorum portant, rationem habet' (HarsColl. 202-203).

- < Pers. sag-bān 'dog-keeper'. - N. 543 (XVII Harsány); P. 218 (XIII/XIV).

491. selv (self/serbi 1603); sälvi (1650) - 1533 selvi (sèlui) 'arcipresso' (ArgAd. 257, ArgR. 213); 1611 self (self) 'abeto, sapin' (RJTMajd. 221); ca. 1630 selvi (selui) 'cupressus' (MontR. 173); 1650 sälvi (salui) 'arcipresso' (CarrR. 289).

Phr. - 1611 servi àgaç (seruí agácc) 'cipresso, albero' (FerrR. 137); 1650 selvi aǵac (selui agag) 'cipresso' (CarrR. 289).

- < Pers. sarw 'cypress-tree; fir-tree'. - N. 545 (XIV); P. 223-24 (XIV).

494. sepet (seped 1641) - 1533 sepet (sepétt) 'corbello, cofano, paniere, pianiera' (ArgAd. 257, ArgR. 213); 1611 sepet (ssepét) 'cassa, forziero, panaro, sportella' (FerrR. 137); ca. 1630 sepet (sepet) 'cista, ladula' (MontR. 173); 1650 sepet (sepet) 'coffa, canestro, cofano' (CarrR. 289).

Der. - 1650 sepetci (sepetgi) 'cestaruolo che le fa [le ceste]' (CarrR. 289) - 1533 sepetcik (sepetgích) 'corbello, cofano, paneruzolo' (ArgAd. 257, ArgR. 213); 1650 sepetcik (sepetgich) 'cofanetto' (CarrR. 289).

Phr. - 1677 sepet santugi $\imath$ (sepet santughi) 'forziere' (MascVoc. 52).

- < Pers. sapad 'basket'. - N. 548 (XII); P. 219 (1514/15).

495. ser (1641) - 1533 ser (ser) 'capo, in aggiammesco, testa' (ArgAd. 258, ArgR. 214); 1650 ser (ser) 'concetto di mente' (CarrR. 289); 1672 ser (szer) 'caput' (HarsColl. 186); 1677 ser (ser) ‘testa, capo’ (MascVoc. 246).

Phr. - 1650 seri meydan [e] ko- (seri ${ }^{\star}$ meidan chorum) 'por la vita in compromesso, a risico' (CarrR. 244).

- < Pers. sar 'head, top, summit'. - N. 549 (XIV); P. 219 (1482).

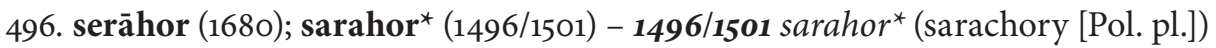
['rodzaj jazdy nieregularnej'] (Constantine of Ostrovica: StachSHET. 498).

- < Pers. sar- axxur' a horse tied at the head of all the rest in a stable; master of the horse'. - N. (-); P. (-).

498. seraser (1641); $\operatorname{seraşer}^{\star}$ (1587/88), sirasir (1650), serasen (1668) - 1587/88 seraşer $^{*}$ ( ${ }^{*}$ jerascher) 'Guldenstuck' (LubAd. 13); 1611 seraser (serasér) 'imbrocato, drappo di seta' (FerrR. 137); ca. 1630 seraser (serasser) 'attalici operis brocato' (MontR. 173); 
1650 sirasir (sirasir) 'broccato d'oro, tela' (CarrR. 295); 1668 serasen (szeraszen) 'auro texta' (IllNém. 194); 1677 seraser (seraser) 'imbroccato d'oro' (MascVoc. 64).

Phr. - $\rightarrow$ 778. diba.

- < Pers. sar-ā-sar 'brocade, embroidery'. - N. (-); P. 220 (2/XV).

499. serasker (1591); saraskar (1675), sarasker (1677) - [Italianised forms such as seraschiere, saraschiere have not been taken into account] - $\mathbf{1 5 3 4}$ serasker ['tytuł dowódcy wojsk'] (Polish source: StachSHET. 510); 1570 "Vi serà coll'armata seraschier (...) il magnifico Piali bassà" (A. Buonrizzo: RelPedF. 155); 1622 serasker (Ser-ascher) 'Feldobrister' (WennStach. 605); 1675 "Sarascar ou Generalissime de l'armee qui est pour l'ordinaire le Grand Vizir" (M. Febvre: ArvAdd. 467); 1677 "Sarasquier, ou General” (de La Magdeleine: ArvAdd.467).

Phr. - 1677 serasker gemisi (seraschier ghiemissi) 'naue dell'ammiraglio' (MascVoc. 104).

- < Pers. sar-'askar 'a general commander of land forces; seraskier'. - N. 549 (XIV); P. (-).

502. serd (168o); sert (1611) - 1611 sert (sért) 'aspro, terribile, crudele' (FerrR. 137); 1641 sert (sert) 'scabroso, rozzo nel tocco' (MolDitt. 376); 1650 sert (sert) 'aspro, scabroso, ruuido’ (CarrR. 290).

Der. - 1611 sertçesine (sertciesiné) 'aspramente' (FerrR. 137) - 1611 sertlik (sertlíc) 'asprezza' (FerrR. 137); 1650 sertlik (sertlich) 'asprezza, ruuidezza, scabrosità', (sertlich ileh) 'aspramente, scabrosamente, horridamente, ritrosamente, ruuidamente' (CarrR. 290).

Phr. - 1650 sert et- (sert ederum) 'acerbire, inasprire' (CarrR. 290).

- < Pers. sard 'cold; disagreeable, unpleasant'. - N. 551 (XIV); P. 221 (XV/XVI).

503. serdar (1591); sardar (1587), zerdar (1611) - 1587 “Sardar, voce Turchesca, da noi Capitano di Gianizari, d'essercito, \& Generale propriamente" (MinHist. unnumbered page); 1598 "Capitan Generale, che Ser-dar chiamano con voce Persiana" (SorOtt. 18); 1611 serdar (serdar) 'capita[n] gen[er]ale' (RJTMajd. 222); 1611 zerdar (szerdár) 'capitan generale d'un esercito o armata' (FerrR. 137); ca. 1630 serdar ${ }^{\star}$ ( ${ }^{\star}$ ferdar) 'adductor, it. conduttiere' (MontR. 173); 1641 serdar (serdara [Pol. gen.]) ['tytuł wodza naczelnego'] (W. Miaskowski: StachSHET. 511-512); 1650 serdar (serdar) 'centurione' (CarrR. 289); 1668 "In every Province the Janizaries have their Serdars [Engl. pl.], who are Collonels" (RycautPSt. 196); 1677 serdar (serdar) 'capitano; generale' (MascVoc. 24, 55).

- < Pers. sar-dār 'general, field-marshal, officer of rank, king's lieutenant'. N. 549 (XIV); P. 221 (2/XVI).

507. sergerdān (1680); zergârdan (1641), sergârdan (1650) - 1641 zergârdan (szerghiardan) 'disuiato' (MolDitt. 126).

Phr. - 1650 sergârdan et- (serghiardan ederum) 'desuiare, subornare', (serghiardan etmech) 'desuiamento' (CarrR. 289) - 1650 sergârdan edici (serghiardan 
edigi) 'desuiatore' (CarrR. 289) - 1650 sergârdan ol- (serghiardan olurum) 'disuiato’ (CarrR. 289).

- < Pers. sar-gardān 'confounded, stupefied, amazed, astonished, distressed'. N. 550 (XIV); P. 222 (XIII/XIV).

511. sermaye (sermaya/sarmaya/sarmaye 1641) - 1533 sermeya (sermeiá) 'il chapitale' (ArgAd. 258, ArgR. 214).

Phr. - 1677 sermaye boz- (sermaie bosmach) 'inuestire il denaro, cioè spendere, o comprare' (MascVoc. 74) - 1677 sermaye çat- (sermaie ciatmach) 'inuestire, cioè dar l'inuestiture, o possesso' (MascVoc. 74).

- < Pers. sar-māya 'the capital of a merchant, stock-in-trade, principal sum'. N. 550 (XIV); P. 223 (1332).

515. sersam/sersem (1680) - 1533 sersem (sersém) 'farnetico, rimbambito' (ArgAd. 258, ArgR. 214).

Der. - 1533 sersemle-/sersenle- (sersenlenérum) 'farnetico', (sersenletterúrum) 'fo farneticare', (sersemlemísc) 'sbalordito' (ArgAd. 258, ArgR. 214).

- < Pers. sarsām 'stupefied; frenzy, delirium'. - N. 551 (XV); P. (-).

519. seyran (1680); $\operatorname{saryan}^{\star}(1560)$ - 1533 seyran (seirán) 'piacere, sollazo, spasso' (ArgAd. 258, ArgR. 215); 1560 "il [= the Sultan] va ainsi Sariana [+ dat.], ou jouer" (PostelTPart. 13); 1677 seyran (seiran) 'sollazzeuole, piaceuole' (MascVoc. 211).

Der. - 1533 seyrancı (seirangí) 'quello che ua a sollazo, chi ua a sollazo, chi ua a spasso' (ArgAd. 258, ArgR. 215) - 1533 seyrancık (seirangích) 'piacere, sollazo, spasso' (ArgAd. 258, ArgR. 215).

Phr. - 1533 seyran et- (seirán edér) 'ua a sollazzo' (ArgAd. 258) - 1533 seyrana git- (seiraná ghider) 'ua a sollazzo' (ArgAd. 258); 1587/88 seyrana* git- ( ${ }^{*}$ seiraua ghider) 'ehr gehet (...) spaciren' (LubAd. 18).

- < Pers. (< Ar.) sayrān 'a walk, drive, or ride for pleasure'. Perhaps a direct borrowing from Arabic. - N. 552 (XIV); P. (-).

520. seyrangâh (1680); sirangâ (1650) - 1650 sirangâ (siranghia) 'loggia' (CarrR. 295).

- < Pers. sayran-gāh 'place of recreation'. - N. (-); P. 224 (XIII/XIV).

521. seysana ( ${ }^{*}$ seiskane [recte seyshane] 1641) - 1533 seysana// seyisana (seisaná) 'charriaggio' (ArgAd. 258, ArgR. 215).

- < Pers. sä'is-xāna 'beast of burden; luggage of a grandee, \&c., when travelling', says-xāna 'baggage-horses employed in war'. - N. (-); P. (-).

522. sezāvār (1680) - 1672 sezavar (sezavar) 'digna' (HarsHaz. 198-199).

- < Pers. sazāvār 'worthy, deserving'. - N. (-); P. 225 (1482).

523. sihirbaz ( ${ }^{*}$ seyrbas [recte sey(i)rbaz] 1641); sehribaz ${ }^{\star}$ (1677), segribaz $^{\star}(1677)$ 1650 sey(i)rbaz (seirbas) 'magha' (CarrR. 291); 1677 sehirbaz (sehirbaz) 'incanto' (MascVoc. 66). 
Der. - 1650 sehirbazcı (sehirbasgi) 'ammaliatore' (CarrR. 288) - 1677 segribazlık (-luk)/sehribazlık (seghribasluch) 'fattura, stregonerie', (sehribaslich) magica arte', (secribaslich) 'negromanzia', (segribaslich) 'ueneficio, incantesimo' (MascVoc. 48 passim).

- 1650 sehirbazla-/sey(i)rbazla- (sehirbaslarum, seirbaslarum) 'ammaliare' (CarrR. 288).

- < Pers. sihrr-bāz ‘enchanter, sorcerer’. - N. 557 (XVII Meninski); P. (-).

526. silihtar (1546); sulahtar (1496/1501), suluftar/suluptar (1548), soluftar (1560), silihdar (1608), silikdar (1672) - 1496/1501 "Czwarta rota (...) zowią ja sulachtar, (...) hetman ich sulachtarbasza" (Constantine of Ostrovica: StachSHET. 519); 1503 selictar (M. Sanudo: SchweickTW. 826); 1534 "tre mila silictari [It. pl.], che cavalcano alla sinistra" (D. de Ludovisi: RelAlb.I 15); 1539 "Silichtar, che porta l'arco \& le frezze’ (RambLibT. 14v); 1548 "el terzo [Sultan’s servant] è `Sulustar (recte probably suluftar), che porta l'arco \& le saette \& la sua spada”; "li Suluptar (...) che caualcano alla banda sinistra del gran Turco" (MenTratt. 121, 151; see the other passage from Menavino quoted below); 1553 "Tra questi sono tre paggi più favoriti, l'uno dei quali si chiama silictar, che porta l'arco, la freccia, e la spada del Gran-Signore" (B. Navagero: RelAlb.I 45); 1560 "selictar ou soluftar qui sont gents de cheual qui marchent a vne des ailes du camp" (PostelTPart. 4); 1608 "der dritt [page] tregt jhm [= to the Sultan] /wie ein Schiltjung / seine Waffen / als Säbel vnd Bogen nach / den nennt man den Silichdar" (SchwSt. 245); 1641 sil(a/i)hdar (*szilchdar) 'scudiere' (MolDitt. 389); 1672 silikdar/silihtar (szilikdar) 'armiger', (szilichtari [Lat. pl.]) 'armigeri' (HarsColl. 172, 236).

Phr. - 1560 Selictaraga (see below); 1668 "the Selictar-Aga, the Kings Swordbearer” (RycautPSt. 28); 1677 silihdar ag்a (silihdar aga) 'scudiero' (MascVoc. 194) - 1496/1501 sulachtarbasza (see above); 1522 "Silictar bassà capo delli silictari, ch'è gente tutta a cavallo" (M. Minio: RelAlb.III 73); 1548 "Dalla banda sinistra quãdo caualca el Re passa vn Capitano chiamato ${ }^{\star}$ Supluphtar (recte Suluphtar) Bascia, con mille et cinque cẽto huomini schiaui del $\operatorname{Re}(. .$.$) la più parte di questi$ Suluphtar sono Christiani renegati" (MenTratt. 148); 156o "Le Capitaine des Selictarlar, appellé Selictarbaßi ou Selictaraga" (PostelTPart. 35); 1645 "kapitan morski Silichtar Basza" (St. Oświęcim: StachSHET. 519).

- 1560 "L'autre [page] luy [= to the Sultan] porte l'arc \& la fleche, \& s'appelle *Bencsilictari (= beg silihtarı)" (PostelTPart. 4).

- < Pers. silāh-dār 'sword-bearer, esquire'. - N. (-); P. (-).

527. sine (1680) - 1641 sine (sinem [prob. + first person poss.]) 'petto, parte dinanzi del corpo' (MolDitt. 303).

- < Pers. sina 'bosom, breast'. - N. 560 (XIV); P. 226 (1482).

528. sinebend (sinebent 1612); sinelbent (1587/88), silebend (1668) - 1587/88 sinelbent (sinel bendt) 'Sten grad oder Stefrich' [wrong meanings?] (LubAd. 56); ca. 1630 
sinelbent (sinelbent) 'pectorale' (MontR. 175); 1650 sinebent (sinebent) 'medaglia' (CarrR. 295); 1668 silebend (szilebend) 'antilena' (IllNém.195); 1677 sinebent (sinebent) 'pettorale del cauallo' (MascVoc. 126).

- < Pers. sina-band 'poitrel of a horse'. - N. (-); P. (-).

530. sipāhi (spahi 1546); spahı (ca. 1520), spaı (1530) - 1473/80 "spacchi (...) soldati che ànno e turchi" (DeiCr. 182); [a great many records of the word found in Italian, French and Polish texts of the $16^{\text {th }}$ century as spac(c)chi, spai resp. spah(i)s, spahys; s(z)pach(i)owie are not mentioned here]; ca. 1520 spahı (spache) 'soldà a cauallo' (LupisON. 1b); 1525/30 spaı (spae) 'soldati' (ITSprAd. 243); 1558 "sotto questo nome de' spahi si comprendono tutti quelli che fanno il mestiere dell'armi a cavallo, percioché spahi non vuol dir altro che soldato a cavallo" (N. Michiel: RelPedF. 112); 1587/88 spahi (spahi) 'Rittersleut' (LubAd. 57); 1598 "I miglior caualieri c'habbia l'Imperio Ottomanno sono gli Spahi” (SorOtt. 12); 1603/1612 spahi (spahi) 'eques, Ritter' (MegThP. 1: 474; MegILT.); 1608 "Spahi der ist so viel als einer vom Adel; ein reysiger" (SchwSt. 247); 1608 "Les Spahi de la porte" (H. de Beauvau: ArvAdd. 479); 1611 spahı (spahe, spahè) [without translation] (RJTMajd. 223); ca. 1630 spai (spai) 'eques leuis armaturae' (MontR. 177).

Phr. - 1611 spailik bildir- (spailíc bildirérum) 'domare' (FerrR. 139).

- < Pers. sipāhī 'military, belonging to an army; horseman, cavalier'. - N. 561 (XIV); P. 226 (1430).

531. sipāriş (1680) - ca. 1630 sipariş (siparis) 'commendatio; mandatum' (MontR. 175).

- < Pers. sipāriš 'recommendation, delegation, commission charge'. - N. 561 (XVII Meninski); P. (-).

[534. sirke (1533); serke/zerke (1611), särke (1650) - 1496/99 sirke* (*stircke) 'essich' (HarffSt. 136); [add.] 1533 sirke (sirchié) 'aceto' (ArgAd. 260, ArgR. 218); 1574 sirke (sirche) 'aceto' (VNAd. 67); 1587/88 sirke (sirkhe) 'Essigk' (LubAd. 57); 1611 serke/zerke (cerkè, zerke, zerkhè) 'vinagre' (RJTMajd. 222); 1611 sirke (sirchié) 'aceto' (FerrR. 1439); ca. 1630 sirke (sirchie) 'acetum' (MontR.175); 1641 sirke (sirchie) 'aceto' (MolDitt. 8); 1650 särke (sarchie) 'aceto' (CarrR. 290); 1677 sirke (sirchie) 'aceto' (MascVoc. 5).

Der. - 1650 serkeli (serchieli) 'acetoso' (CarrR. 290).

Phr. - 1641 sul sirke (suli sirchie) 'aceto mescolato con acqua' (MolDitt. 8); 1650 sult serke (suli serchie) 'aceto con acqua' (CarrR. 290).

- 1641 gül sirkesi (ghiul sirkiesi) 'aceto rosato' (MolDitt. 13-14); 1677 gül sirkesi (ghiul sirchiesi) 'aceto rosato' (MascVoc. 6).

- 1650 serkeli et- (serchieli ederum) 'acetire' (CarrR. 290) - 1650 serkeli ol(serchieli olurum) 'acetirsi' (CarrR. 290).

- Turkic word surely related to Pers. sirka 'vinegar', but there is controversy about the origin and direction of borrowing of this lexical type (TMEN 3: 246-247; ErenTDES 370). - N. 561 (XI); P. 227 (1332)]. 
536. sitare (sitara 1533) - [add.] 1533 sitara (sitará) 'felicità, fortuna buona, uentura, sorte' (ArgAd. 260, ArgR. 219); 1611 (eyi) sutara (ei sutarā) 'bien auenturança' (RJTMajd. 224); 1650 sutara (sutara) 'sorte, fortuna' (CarrR. 302).

Der. - 1533 sitaralı (sitaralí) 'felice, fortunato, auenturato in buona parte, uenturato (ArgAd. 26o, ArgR. 219); 1611 sutaralı (sutaralí) 'fortunato, auuẽturato' (FerrR. 139) - 1533 sitarasız (sitarasís) 'suenturato' (ArgAd. 260, ArgR. 219); 1611 sutarasız 'desdicha, desuentura' (RJTMajd. 224); 1611 sutarasız (suttará sís) 'disgratiato, maluẽturato' (FerrR. 139).

- < Pers. sitāre 'star'. - N. 562 (XV); P. 228 (XIII/XIV).

539. siyah (1641); siya (1611) - 1533 (siách) 'morello, cauallo; nero, di panni et drappi et tele' (ArgAd. 260, ArgR. 219); 1611 siya (sihá) 'negro' (FerrR. 139); ca. 1630 siya ( ${ }^{*}$ scia) 'niger' (MontR. 175); 1677 siyah (sijah) 'nero, negro' (MascVoc. 104).

Der. - 1677 siyahlik (siiahlich) 'nerezza, negrezza' (MascVoc. 104).

Phr. - 1533 siyah et- (siách etterúrum) 'fo innegrire' (ArgAd. 260, ArgR. 219); 1611 siya et- (sihá edérum) 'annegrire' (FerrR. 139); 1650 siyah et- (sijah ederum) 'annegrire' (CarrR. 296).

- < Pers. siyāh 'black'. - N. 563 (XIV); P. 228 (1445).

540. sohta (1603); cohda // çohda (?) (1587/88), sufita (1611), softi (ca. 1630) - 1587/88 "der Studiosus oder Dschochda" (LubAd. 59); 1598 "due mila Softi [It. pl.], che sono scolari” (SorOtt. 2); 1608 "ein Schuler oder Dschochda" (SchwSt. 246); 1611 sufita (suffitá) 'fuoruscito, bandito, sbandito di campagna' (FerrR. 139); ca. 1630 softi (softi) 'castus, religiosus, sapientiae studens' (MontR. 176); 1650 softa (softa) 'conuerso de frati' (CarrR. 296); 1668 "a poor Student called by the Turks a Softa" (RycautPSt. 20); 1677 softa (softa) 'scuolare' (MascVoc. 189).

- < Pers. soxta 'burnt; student'. - N. 564-565 (XV); P. 229 ('burnt' 1430).

543. surnapa ( ${ }^{*}$ surnofa 1/XV); sarnapa (1557) - 1533 zurnapa (xurnapá) 'giraffa' (ArgAd. 300, ArgR. 277); 1553/55 "ein frembdt thyr haben wir gesehn (...), haist man Serapha vnd auff turkish surnapa" (DernBab. 53); 1557 sarnapa (sarnapa) ['żyrafa'] (E. Otwinowski: StachSHET. 501; other attestations of the form sarnapa from Polish writers date back to 1569 and 1633: id.); ca. 1630 surnaba // sürnaba (surnaba) 'camelopardus' (MontR. 178); 1677 zurnapa (szurnapa) 'giraffa' (MascVoc. 56).

- < Pers. surnāpā, zurnāpā, zurnapā 'cameleopard'. - N. (-); P. (-).

550. şadurvan (1641) - 1533 şadırvan (sciaderuán) 'fontana che getta l'acqua in alto; zampillo' (ArgAd. 265, ArgR. 227).

Der. - 1533 şadırvancı (sciaderuangí) 'chi le [= fountains called şadırvan] fa' (ArgAd. 265, ArgR. 227).

- < Pers. šādurbān, šādurwān 'large veil, curtain; cornice; eaves, projecting roof'. - N. 582 (XVII Meninski); P. (-). 
552. şah (1544); şaha (ca. 1630) - 1545 "Quando [the Sultan] fa l'intrata in Prusia o in Andrinopoli i Giudei con voci adulatorie l'accompagnano cantando, o sanna benedetto sia il Signore nostro Sultan Suleiman Sah" (BassR. 78); 1584 "Schach heist König" (SchwSt. 249); ca. 1630 şaha (saha, sahha) 'rex Persar(um)' (MontR. 180); 1633 şah (szach) ['tytuł dawnych władców perskich'] (S. Twardowski: StachSHET. 545).

Der. - 1587/88 şahluk (schach luck) 'Konigkreich’ (LubAd. 9).

See $\rightarrow$ 559. şahzade, 948. şahbaz.

- < Pers. šāh 'king, sovereign, emperor'. - N. 582 (XI); P. 233 (XIII/XIV).

556. şahi (1591) - 1587/88 şahi (schahi) 'eine Muntz von 8 Aspern' (LubAd. 58); 1608 "Schahi ein silberne Müntz / (...) deren einer thut achthalben Asper" (SchwSt. 250).

- < Pers. šā $\bar{c}$ 'royal; imperial dignity; name of a coin'. - N. (-); P. 234 (1489).

557. şahin (1603); şain (1677) - 1533 şahin (sciachín) 'falcone pelegrino’ (ArgAd. 266, ArgR. 227); 1650 şahin (sciahin) 'arditello, arditetto' (CarrR. 305); 1672 şahin (sahin) 'falco' (HarsColl. 425); 1677 şain/şahin (sciain) 'falcone', (sciahin) 'sparuiere, vccello' (MascVoc. 47, 218).

- < Pers. šāhīn 'royal white falcon', šăhin 'excellent falcon'. - N. 582 (XIV); P. 234 (XIV).

559. şahzade (1591) - 1677 şehzade (sceh-szadè) 'principe' (MascVoc. 137).

Phr. - 1668 "Schachzadeler Agasi, or the Eunuch to whose charge is committed the Royal Progeny" (RycautPSt. 37).

- < Pers. šāh-zāda 'king's son'. - N. (-); P. 234 (1445).

560. şakird (şayirt 1455/56); şegirt (1533), şeyirt (1611), şehird (1677) - 1533 şegirt (sceghírt, sceghírtt) 'discepolo, factore, discipulo, scolare' (ArgAd. 266, ArgR. 229); 1611 şeyirt (scieírt) 'discepolo’ (FerrR. 143); ca. 1630 şagirt/şegirt (sagirt, saghirt, sciegirt) 'discipulus' (MontR. 180); 1677 şeyirt/şayird/şehird (sceirt) 'discepolo', (sciaird, scehird) 'scuolare' (MascVoc. 39, 189, 194).

- < Pers. šāgird 'scholar, student, apprentice, disciple, pupil'. - N. 583 (XIII); P. 234 (1430).

561. şal (1641) - 1533 şal (sciàl) 'chamiscia de' chaualli' (ArgAd. 266, ArgR. 227).

- < Pers. šāl 'shawl or mantle, made of very fine wool; coarse mantle of wool and goats' hair; horse-cloth'. - N. 583 (XVII Meninski); P. 234 (XV).

562. şalvar (1680); şalfar (1611) - 1533 şalvar (scialuár) 'chalzoni grandi oue a chammino si mettono le ueste' (ArgAd. 266, ArgR. 228); 1611 şalfar (schalfhàr) 'weite hosen wür (!) frauen' (RJTMajd. 224).

- < Pers. šalwār 'inner breeches, drawers reaching to the feet; sailor's or traveller's trousers'. - N. 584 (XV); P. (-). 
563. şamdan (1603); sandan (1587/88), şamedan (1611), şämdar (1611) - 1533 şamdan (sciamdán) 'chandelliere' (ArgAd. 266, ArgR. 228); 1587/88 sandan (sandan) 'Leuchter' (LubAd. 58); 1611 şamedan (schamedan) 'candilero' (RJTMajd. 224); 1611 şämdar (sciemdár) 'candeliere' (FerrR. 143); ca. 1630 şamdan (samdan) 'candelabrum' (MontR. 180).

Der. - 1533 şamadancı (sciamadangí) 'chandelleraro' (ArgAd. 266, ArgR. 228). - < Pers. šam'dān 'candlestick, chandelier'. - N. 584 (XVI Argenti); P. (-).

565. şayed (1603); şaat (1533) - 1533 şaat (sciaát) 'forse' (ArgAd. 266, ArgR. 227).

- < Pers. šâyad 'let it be; may be, perhaps'. - N. 587 (XIV); P. 235 (1430).

567. şeftali (şefteli 1603); şiftali/şifteli (1611), çefteli (1611) - 1533 şefteli (sceffttelj́) 'pescha' (ArgAd. 266, ArgR. 229); 1587/88 şeftalü/şeftali (scheftalu) 'Pfirsich'; (scheftali) 'Kus' (LubAd. 58); 1611 şiftali/şifteli (schifthali) 'persicos'; (schiftheli) 'beso' (RJTMajd. 225); [add.] 1611 çefteli (cieftelí) 'bacio; persico, frutto' (FerrR. 144); ca. 1630 şefteli (scefteli) 'persicũ malum' (MontR. 181).

Phr. - 1533 şefteli agacı (sceffttelí aghaggí) 'pesco' (ArgAd. 266, ArgR. 229); 1611 çefteli àgaç (cieftelí agácc) 'persico, albero' (FerrR. 144); ca. 1630 şefteli ag̉ac (sceffteli agag) 'persica arbor' (MontR. 181) - 1611 tüysiz çefteli (tui sís cieftelí) 'noce persico' (FerrR. 144) - $\rightarrow$ 810. gülişefteli.

- < Pers. šaftālū 'peach (rough plum)'. - N. 588 (XV); P. 237 (1445).

569. şehir (şar 1533); şeer (1611) - 1574 şar (ssar) 'città' (VNAd. 67); 1575 seher (seher) 'ville; ciuitas' (PostelInstr.); 1587/88 şeher (scheher) 'Stadt' (LubAd. 58); 1611 şeher (scheher, scheherr) 'ciudad, la tierra' (RJTMajd. 225); 1611 şeher (sciehér) 'cità' (sic) (FerrR. 144); ca. 1630 şeher (seher) 'vrbs; circuitus moenium' (MontR. 181); 1650 sehir/şeher (sehir, sceher) 'città' (CarrR. 306); 1677 şehr (scechr) [in several placenames], (scehri [+ poss.]) 'città' (MascVoc. 29).

Der. - 1611 şeherli (scieherlí) 'citadino’ (sic) (FerrR. 144); ca. 1630 şeherli (seherli) 'ciuis; popularis' (MontR. 181); 1650 şeherli (sceherli) 'terrazzano' (CarrR. 306); 1677 şehrili/sceherli (scehrili) 'cittadino', (sceherli) 'patrizio; urbano, chi è della città' (MascVoc. 29, 120, 276) - 1650 sehirlik/şeherlik (sehirlik = šhrlk) 'cittadinanza' (CarrR. 306).

Phr. - 1530 "il sarameni, cioè il fattor della città qual pagha li iannizzerotti, le donne del serraglio, garzoni, pupilli et fabriche" (T. Mocenigo: RelPedF. 44); 1539 "vno Saraemini, che è come Proueditor di commun, che fa conciar le strate in Costantinopoli, \& cosi per camino quando il Signor escie fuori alla guerra: \& similmente ha cura delle fabriche publice, fontane, \& acquedutti" (RambLibT. 20r); 1557 "Al seremin, per le spese delle strade della città e fuori, e dei serragli delle donne e dei garzoni" (A. Erizzo: RelAlb.III 130); 1560 "Il y a le ssaremin ou maistre de œuures qui prend garde aus edifices tant publicques cõme particulieres" (PostelRepT. 126) - 1611 şeer oğlanı (scheèr oglani) 'citadino' (RJTMajd. 225); 1641 sehir og̉lanı (sehir oglani) 'scaltrito, accorto' (MolDitt. 377) - $\rightarrow$ 88. çirkef, 127. duvar.

- < Pers. šahr, šār 'city, town'. - N. 588 (XIII); P. 237 (1291-1312). 
572. şeker (şeher [? uncertain reading] 1553); şiker (1611), zukâr (1641), şäker (1650), säkär (1650) - 1533 şeker (scecchiér) 'zucchero' (ArgAd. 267, ArgR. 229); 1587/88 şeker (scheker) 'Zucker' (LubAd. 58); 1611 şeker (scheker, schekèr) 'asucaro' (RJTMajd. 225); 1611 şiker (scichér) 'zuccaro' (FerrR. 144); ca. 1630 şeker (siecher) 'saccarum' (MontR. 181); 1641 zukâr (szukiar) 'zuccaro' (MolDitt. 494); 1650 säkär (sachar) 'zucharo' (CarrR. 288); 1677 şeker (scechier) 'zuccaro' (MascVoc. 280).

Der. - 1533 şekerci (scecchiergí) 'spetiale di confectioni, li spetiali di zuchero' (ArgAd. 267, ArgR. 230).

- 1677 şekerle- (sciechierleme) 'confezioni' (MascVoc. 32).

Phr. - 1533 nöbet şeker (nobétt scecchiér) 'zuchero chandito' (ArgAd. 241, ArgR. 184) - $\rightarrow$ 809. gülbeşeker.

- 1533 şeker işi (scecchiér jscì) 'confectione' (ArgAd. 267, ArgR. 230); 1650 şäker ışı (sciacher asci) 'boccellato, ciambella, biscottello' (CarrR. 307) - 1611 şeker kamışı (scheker camischschi) 'camnia de zucaro' (RJTMajd. 225) - 1611 şiker kişniş (scichiér chiscnísc) 'confetti di zuccaro' (FerrR. 110) - 1533 şeker penyir (scecchiér pegnír) 'pennito' (ArgAd. 267, ArgR. 230).

- ca. 1630 şekerci başı (sekergi bassi) 'pharmacopola' (MontR. 181).

- 1677 şekerleme kutusı (scechierlemè cutussi) 'scatola di confetti' (MascVoc. 185).

- < Pers. šakar, šakkar 'sugar'. - N. 588 (XI); P. 238 (XIV).

575. şikâr (1641) - 1533 şikâr (scicchiár) 'preda' (ArgAd. 268, ArgR. 231).

Der. - 1677 şikârlı (scichiarli) 'rapinoso' (MascVoc. 150).

- 1677 şikârla- (scichiarlamach) 'rapire', (scichiarlaian) 'rapitore' (MascVoc. $150)$.

- < Pers. šikār 'prey, game; chase, hunting'. - N. 592 (XIV); P. 240 (XIII/XIV).

578. şimşir (çimşir 1603) - 1533 şimşir (scimscír) 'bossolo, arboro' (ArgAd. 268, ArgR. 231); 1611 çimşir (tschimschir) 'eleboro' (RJTMajd. 46).

- < Pers. šimšād 'any tall and upright tree; box-tree', šimšār 'box-sprig'. N. 592 (çimşir XVIII [!]); P. 241 (şimşâd 2/XV); TETTL 1: 519 (çimşir 1640).

579. şira (1641) - 1533 şira /l şıra (scirá) 'mosto’ (ArgAd. 268, ArgR. 231).

- < Pers. šìra 'new wine'. - N. 591 (XI); P. (-).

580. şirin (1641) - 1533 şirin (scirín) ‘bello’ (ArgAd. 268, ArgR. 232); 1677 şirin (scirin) 'soaue, dolce' (MascVoc. 208).

Der. - 1533 şirinlik (scirinlích) 'belleza' (ArgAd. 268, ArgR. 232); 1677 şirinlik (scirinlich) 'soauità, dolcezza' (MascVoc. 208).

Phr. - 1650 şirin et- (scirin ede[rum]) 'raddolcire, mitigare la collora' (CarrR. 309) - 1650 şirin ol- (scirin olmisc) 'addolcito, raddolcito' (CarrR. 309).

- < Pers. širinn 'milky, sweet; pleasant, gentle; gracious, affable'. - N. 592 (XII);

P. 241-42 (XIII/XIV). 
581. şişe (1533); şişä (1611), şice (1603), çiçe (ca. 1630), şişek/şişik (?) (1650) - [add.] 1533 şişe (sciscé) 'guastada' (ArgAd. 268, ArgR. 232); 1587/88 şişe (schische) 'Fensterglas' (LubAd. 59); 1603/1612 şice (schigè) 'vitrum' (MegThP. 2: 719; MegILT.); 1611 şişe (schische, schisché) 'eng[o]stara, ambulla, bouteille' (RJTMajd. 225); 1611 şişä (scisciá) 'caraffa' (FerrR. 145); ca. 1630 çiçe (chichie) 'ampulla' (MontR. 71); 1650 şişe/şişik (?) (sciscich = šyšh) 'amola, caraffa, inghistara, ampolla, fiascho', şişek (sciscech) 'ampolla' (CarrR. 310).

Phr. - 1611 kan alacak şişä (cán alagiác scisciá) 'ventose da cauar sangue' (FerrR. 145).

- < Pers. šiša 'glass, bottle, flask, phial, cup, caraff' (see ErenTDES 388, TMEN 3: 328-330). - N. 593 (XIII); P. 242 (1445).

\section{Abbreviations}
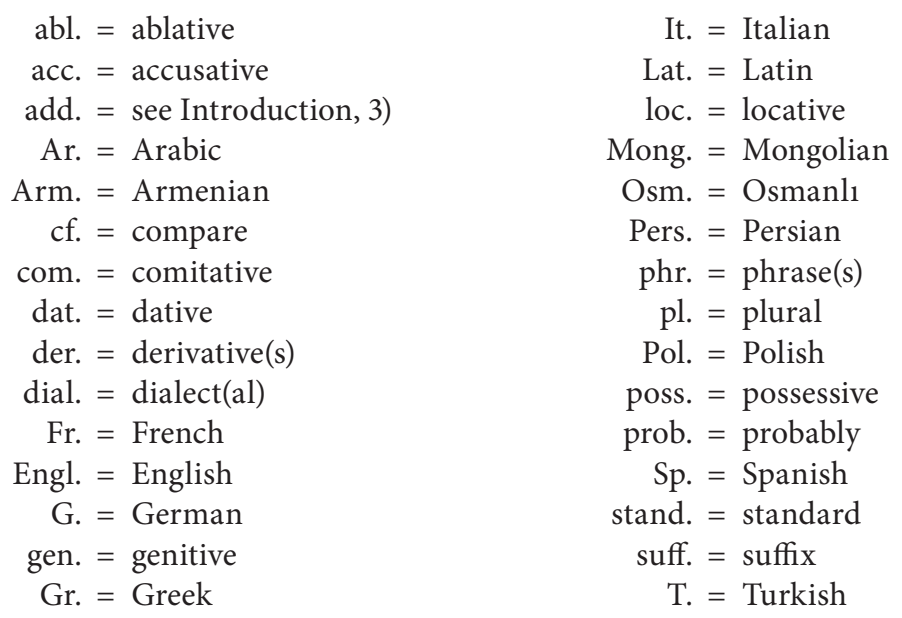

\section{References}

ArgAd. = Adamović M. (ed.). 2001. Das Türkische des 16. Jahrhunderts. Nach den Aufzeichnungen des Florentiners Filippo Argenti (1533). Göttingen.

ArgR. = Rocchi L. (ed.). 2007. Ricerche sulla lingua osmanli del XVI secolo. Il corpus lessicale turco del manoscritto fiorentino di Filippo Argenti (1533). Wiesbaden.

ArvAdd. = Arveiller R. 1999. Addenda au FEW XIX (Orientalia). Tübingen.

BassR. $\quad=$ Rocchi L. 2006. Esotismi nell'italiano cinquecentesco. Il corpus alloglotto dell'opera di Luigi Bassano da Zara. - Rivista Italiana di Linguistica e Dialettologia 8: 57-84.

BodrogPVCC $=$ Bodrogligeti A. 1971. The Persian vocabulary of the Codex Cumanicus. Budapest.

BVenON. = Venetiano B. 1580. Opera Nova de Vocaboli Turcheschi, \& Gregheschi (...). Venezia. 
CardonaVOr. = Cardona G.R. 1969. Voci orientali in avvisi a stampa romani del '500. Lingua Nostra 30: 5-9.

CarrR. = Rocchi L. (ed.). 2011. Il dizionario turco-ottomano di Arcangelo Carradori (1650). Trieste.

ClausonED = Clauson G. 1972. An etymological dictionary of pre-thirteenth century Turkish. Oxford.

COED = The compact Oxford English dictionary. $1991^{2}$. Oxford.

DankoffArm. = Dankoff R. 1995. Armenian loanwords in Turkish. Wiesbaden.

DankoffEÇGl. $=$ Dankoff R. 1991. An Evliya Çelebi glossary. Unusual, dialectal and foreign words in the Seyahat-name. Boston.

DeiCr. = Dei B. 1984. "Cronica", a cura di R. Barducci. Firenze.

DELI = Cortelazzo M., Zolli P. 1979-1988. Dizionario Etimologico della Lingua Italiana. [vol. 1-5]. Bologna.

DernBab. = Babinger Fr. (ed.). 1923. Hans Dernschwams Tagebuch einer Reise nach Konstantinopel und Kleinasien (1553/55). München, Leipzig.

DValCard. = Cardini C. (ed.). 2001. La Porta d'Oriente. Lettere di Pietro Della Valle: Istanbul 1614. Roma.

ErenTDES = Eren H. 1999. Türk Dilinin Etimolojik Sözlüğü. Ankara.

ÈSTJa = Sevortjan Ė.V. et al. 1974-2003. Ėtimologičeskij Slovar' Tjurkskih Jazykov. [vol. 1-7]. Moskva.

FerrR. $\quad=$ Rocchi L. (ed.). 2012. Il "Dittionario della Lingua Turchesca" di Pietro Ferraguto (1611). Trieste.

GeorgHeff. = Heffening W. (ed.). 1942. Die türkischen Transkriptionstexte des Bartholomaeus Georgievits aus den Jahren 1544-1548. Leipzig.

GIITR. = Rocchi L. (ed.). 2016. Il glossario italo-turco contenuto in codice fiorentino del XVI secolo. - Zeitschrift für romanische Philologie 132(4): 1049-1064.

GUngSt. = Stein H. (ed.). 1995/6, 1997. Das Türkische Sprachgut im “Tractatus de Moribus, Condictionibus et Nequicia Turcorum” (1481) des Georg von Ungarn. - [1] Archivum Ottomanicum 14: 39-78; [2 (Lautgeschichtliches)] Archivum Ottomanicum 15: 89-118.

GülensoyKBS = Gülensoy T. 2011. Türkiye Türkçesindeki Türkçe Sözcüklerin Köken Bilgisi Sözlüğ̈̈. [vol. 1-2]. Ankara.

HaimFarh. = Haim S. 1953. Farhang Moaser Persian-English dictionary. Tehran.

HarffSt. = Stumme H. 1914. Das Arabische und das Türkische bei Ritter Arnold von Harff. - (n. ed.). Festschrift für Ernst Windisch. Lepzig: 127-137.

HarsColl. = Nagy de Harsány J. 1672. Colloquia Familiaria Turcico Latina (...). Coloniae Brandeburgicae.

HarsHaz. = Hazai Gy. (ed.). 1973. Das Osmanisch-Türkische im XVII. Jahrhundert. Untersuchungen an den Transkriptionstexten von Jakab Nagy de Harsány. Budapest.

IllNém. = Németh J. (ed.). 1970. Die türkische Sprache in Ungarn im siebzehnten Jahrhundert. [Edition of the Illésházy-Codex (1668)]. Amsterdam, Budapest.

ITSprAd. = Adamović M. (ed.). 1975. Ein italienisch-türkisches Sprachbuch aus den Jahren 1525-1530. - Wiener Zeitschrift für die Kunde des Morgenlandes 67: 217-247. [A copy of $\rightarrow$ LupisON with a few variations].

JunkerAlaviW. $=$ Junker H.F.J., Alavi B. 1965. Wörterbuch Persisch-Deutsch. Leipzig.

LettBomb. = Bombaci A. (ed.). 1939/1949. Una lettera turca in caratteri latini del dragomanno ottomano Ibrāhīm al veneziano Michele Membre (1567). - Rocznik Orientalistyczny 15: 129-144. 
LubAd. = Adamović M. (ed.). 1977. Das osmanisch-türkische Sprachgut bei R. Lubenau. München.

LupisON. = Lupis P. ca.1520. Opera Nova de M. Pietro Lupis Valentiano. La qual insegna a parlare Turchesco. Ancona.

MachDisc. = Machiavelli N. 1984. Discorsi sopra la prima deca di Tito Livio. Premessa al testo e note di Giorgio Inglese. Milano.

MancT. = Mancini M. 1990. Turchismi a Roma e a Venezia. - Poli D. (ed.). Episteme. In ricordo di Giorgio Raimondo Cardona. Roma: 75-112.

MascVoc. = Mascis A. 1677. Vocabolario Toscano e Turchesco. Firenze.

MegILT. = Megiser H. 1612. Institutionum Linguae Turcicae Libri Quatuor. Lipsiae.

MegThP. = Megiser H. 1603. Thesaurus Polyglottus vel Dictionarium Multilinguae (...). Francofurti ad Moenum.

MenTratt. = Menavino G.A. 1548. Trattato de costumi et vita de Turchi. Firenze.

MinHist. = Minadoi G. 1587. Historia della guerra fra Turchi et Persiani. Roma.

MolDitt. $\quad=$ Molino G. 1641. Dittionario della lingua Italiana, Turchesca. Roma.

N. $\quad$ = Nişanyan S. 2009. Sözlerin Soyağacı. Çağdaş Türkçenin Etimoljik Sözlüğü. [ $4^{\text {th }}$ edition]. İstanbul.

NicQLivr. = de Nicolay N. 1568. Les quatre premiers livres des navigations et pérégrinations orientales. Lyon.

P. $\quad=$ Pomorska M. 2013. Materials for a historical dictionary of New Pérsian loanwords in Old Anatolian and Ottoman Turkish from the $13^{\text {th }}$ to the $16^{\text {th }}$ century. Kraków.

PalBern. = Palerne J. 1991. D’Alexandrie à Istanbul. Pérégrinations dans l'Empire Ottoman 1581-1583. [Introduction et annotations d'Yvelise Bernard]. Paris. [Palerne's manuscript dates back to 1584].

PalPD. = Palerne J. 1606. Peregrinations (...). Plus est adiousté un petit dictionaire en langage françois, italien, grec vulgaire, turc, moresque, ou arabesque, \& esclauon (...). Lyon. [See PalBern].

PaszkStach. = Stachowski M. 2013. Marcin Paszkowski’s Polish and Turkish dictionary (1615). - Studies in Polish Linguistics 8: 45-55.

PostelInstr. = Postel G. 1575. Instruction des motz de la langue turquesque les plus communs, put as an introduction (without page numbering) to the book by the same author Des Histoires orientales et principalement des Turkes ou Turchiques (...). Paris.

PostelRepT. = Postel G. 1560. De la Republique des Turcs (...). Poitiers.

PostelTPart. = Postel G. 1560. La tierce partie des Orientales Histoires (...). Poitiers.

RambLibT. = Ramberti B. 1539. Libri tre delle cose de Turchi. Venezia.

RamNav. = Ramusio G.B. 1550. Delle Navigationi et Viaggi. Venezia.

RäsänenVW. = Räsänen M. 1969. Versuch eines etymologischen Wörterbuchs der Türksprachen. Helsinki.

RelAlb.I = Alberi E. (ed.). 1840. Relazioni degli ambasciatori veneti al Senato. [Serie 3, vol. 1]. Firenze.

RelAlb.III = Alberi E. (ed). 1855. Relazioni degli ambasciatori veneti al Senato. [Serie 3, vol. 3]. Firenze.

RelPedF. = Pedani-Fabris M.P. (ed.). 1996. Relazioni di ambasciatori veneti al Senato. Volume XIV. Costantinopoli - Relazioni inedite. Padova.

RJTMajd. = Majda T. (ed.). 1985. Rozwój języka tureckiego w XVII wieku (rękopis z 1611 r., ze zbiorów Biblioteki Uniwersyteckiej we Wrocławiu, sygn. M. 1529). Warszawa. 
RycautPSt. = Rycaut P. 1668. The present state of the Ottoman Empire (...). London.

SchwSt. = Stein H. (ed.). 1987. Das türkische Sprachmaterial in Salomon Schweiggers Reisebuch (1608). - Acta Orientalia Academiae Scientiarum Hungaricae 41.2: 217-266.

SchweickOsm. $=$ Schweickard W. 2011. Osmanismen in den europäischen Sprachen. Vorüberlegungen zu einem vergleichenden historischen Wörterbuch. - Lexicographica 27: 221-239.

SchweickStrat. $=$ Schweickard W. 2011. La stratificazione cronologica dei turchismi in italiano. - La Lingua Italiana. Storia, Strutture, Testi 7: 9-16.

SchweickTurk. $=$ Schweickard W. 2013. Turkisms in Italian, French and German (Ottoman period, 1300-1900). A historical and etymological dictionary. [http://www. uni-saarland.de/lehrstuhl/schweickard/turkisms.html].

SchweickTW. = Schweickard W. 2014. Türkische Wortgeschichte im Spiegel europäischer Quellen. - Zeitschrift für romanische Philologie 130.2: 815-832.

SorOtt. = Soranzo L. 1598. L'Ottomanno. Ferrara.

SpandSath. = Spandugnino Patritio Constantinopolitano Th. 1890. De la origine de li imperatori ottomani, ordini della corte, forma del guerreggiare loro, religione, rito, et costumi della natione. - Sathas C.N. (ed.). Documents inédits relatifs à l'histoire de la Grèce au moyen âge. [vol. 9]. Paris: 133-261.

StachSHET. = Stachowski S. 2014. Słownik historyczno-etymologiczny turcyzmów w języku polskim. Kraków.

StachWMong. = Stachowski M. 2012. Written Mongolian čamča 'shırt' and its etymological counterparts in Europe. - Hyytiäinen T. et al. (eds.). Per Urales ad Orientem. Iter polyphonicum multilingue. Helsinki: 445-451.

StanWSPA = Stanisławski J. 1994. Wielki słownik polsko-angielski/The great PolishEnglish dictionary. Warszawa.

Steingass = Steingass F. 1892. A comprehensive Persian-English dictionary. London.

TETTL= Tietze A. 2002, 2009. Tarihi ve Etimolojik Türkiye Türkçesi Lugatı/Sprachgeschichtliches und etymologisches Wörterbuch des Türkei-Türkischen. [vol. 1: A-E]. İstanbul,Wien; [vol. 2: F-J]. Wien.

TietzePAbl. = Tietze A. 1964. Persische Ableitungssuffixe im Azerosmanischen. - Wiener Zeitschrift für die Kunde des Morgenlandes 59-60: 154-200.

TLIO = (n. ed.). 1997-. Tesoro della Lingua Italiana delle Origini. [http://www.tlio. ovi.cnr.it/TLIO/].

TMEN = Doerfer G. 1963-1975. Türkische und mongolische Elemente im Neupersischen. [vol. 1-4]. Wiesbaden.

TS = (n. ed.). 1963-1977. XIII. yüzyıldan beri Türkiye Türkçesiyle yazılmış kitaplardan toplanan tanıklariyle tarama sözlüğ̈̈. [vol. 1-8]. Ankara.

UrbanTTEt. = Urban M. 2015. The treatment of Turkic etymologies in English lexicography. Lexemes pertaining to material culture. Kraków.

VNAd. = Adamović M. (ed.). 1976. Vocabulario nuovo mit seinem türkischen Teil. Rocznik Orientalistyczny 38: 43-69.

WennStach. = Stachowski M. (ed.). 2015. Osmanisch-Türkische Appellativa im Reisebuch von Adam Wenner (1622). - Ragagnin E., Wilkens J. (eds.). Kutadgu Nom Bitig. Festschrift für Jens Peter Laut zum 60. Geburtstag. Wiesbaden: 593-607. 
